\title{
Relationship Between Masked Hypertension Measured by Ambulatory Blood Pressure Monitoring and Left Ventricular Global Longitudinal Strain
}

\section{Ming Fu}

Guangdong Provincial People's Hospital

Xiangming Hu ( $\sim$ hxmgdcz@163.com )

Guangdong Provincial People's Hospital https://orcid.org/0000-0003-2474-0858

\section{Shixin Yi}

Guangdong Provincial People's Hospital

\section{Shuo Sun}

Guangdong Provincial People's Hospital

\section{Ying Zhang}

Guangdong Provincial People's Hospital

\section{Yingqing Feng}

Guangdong Provincial People's Hospital

\section{Yingling Zhou}

Guangdong Provincial People's Hospital

\section{Qingshan Geng}

Guangdong Provincial People's Hospital

\section{Haojian Dong}

Guangdong Provincial People's Hospital

\section{Original Article}

Keywords: masked hypertension, global longitudinal strain, myocardial damage, nonlinear relation

Posted Date: February 17th, 2021

DOI: https://doi.org/10.21203/rs.3.rs-201580/v1

License: (c) (1) This work is licensed under a Creative Commons Attribution 4.0 International License. Read Full License 


\section{Abstract}

Masked hypertension (MHT), as an independent clinical entity, its subclinical cardiac dysfunction can be early detected by left ventricular global longitudinal strain (GLS). Yet the relationship between MHT and GLS is still unclear. Therefore, we tried to conduct a community-based cross-sectional study to define this relationship. A total of 308 consecutively enrolled participants from Dongguan, China, were divided into non-hypertension (NHT) and MHT groups. Baseline characteristics were recorded, and 2-dimentional speckle-tracking echocardiography and 24-hour ambulatory blood pressure monitoring were performed. Univariate and multivariate linear regression analyses were used to assess the associations between MHT and GLS, and the dose-response curve was plotted to demonstrate their relationship. The mean age of the NHT and MHT groups was 57 and 59 years, respectively. Signs of left ventricular diastolic function, $\mathrm{E} / \mathrm{A}(1.01 \pm 0.26$ vs $0.86 \pm 0.23, \mathrm{P}<0.001)$ and $\mathrm{E} / \mathrm{e}^{\prime}(8.58 \pm 2.02$ vs $11.70 \pm 3.37, \mathrm{P}<0.001)$ were reduced in the MHT group while those of the NHT group were nearly normal. The MHT group also showed a significantly lower ('worse') GLS than NHT $(-15.79 \% \pm 2.81 \%$ vs $-19.62 \% \pm 1.67 \%$ vs , $\mathrm{P}<0.001)$ while left ventricular ejection fraction (LVEF) did not differ between the groups. Worse GLS was independently and significantly associated with MHT both in univariate (odds ratio [OR]: 1.97, $P<0.001$ ) and stepwise multivariate regression analysis (OR: 1.99, P < 0.001). Further analysis showed adjusted nonlinear correlation between MHT and GLS. Our study described the relationship between MHT and GLS and concluded that $-14 \%$ of GLS as the cut-off value reflected MHT-associated myocardial injury before LVEF decreases.

\section{Introduction}

Masked hypertension (MHT) is characterized by normal blood pressure (BP) readings in the office and increased out-off-office BP readings. With the wide use of ambulatory blood pressure monitoring (ABPM) and home blood pressure monitoring (HBPM), this phenomenon has been increasing in routine health care. The prevalence of MHT in the general population ranges from 8-30\% [1]. Unlike office hypertension, the target organ damage caused by $\mathrm{MHT}$ is insidious. Recent comparative studies have shown that MHT has $67 \%$ higher risk of all-cause mortality and a $71 \%$ higher risk of fatal and nonfatal cardiovascular events compared with normotensive individuals $[2,3]$. Early recognition of minor cardiac structural damage in patients with $\mathrm{MHT}$ would be of great significance for timely treatment and improved prognosis of these patients [4].

Since most community residents lack routine health screening, most patients with MHT are not discovered until they develop symptoms caused by persistent hypertension, at which time there has already been significant cardiac structural remodeling and eventually irreversible decrease in left ventricular ejection fraction (LVEF) [5]. Recently, assessment of global longitudinal strain (GLS) with the use of speckle-tracking echocardiography technique has shown the ability to recognize minor damage in cardiac function before the decrease in LVEF. However, the association between MHT and impaired GLS has not yet been extensively discussed. Furthermore, it is also unknown whether their relation is affected 
by common hypertension-related factors including individual behavior and co-existing diseases. Therefore, we launched a community-based population screening cohort to try to address this issue.

\section{Materials And Methods}

All participants gave orally informed consent for study participation. This study was approved by the Ethical Committee of Guangdong Provincial People's Hospital, and was conducted in accordance with the principles of the Declaration of Helsinki II.

\section{Study population}

This study was designed as a retrospective study in the population that had participated in the high-risk cardiovascular screening for chronic-care management in Liaobu Town, Dongguan, in 2012. In this study, the inclusion criteria were as follows: 1 ) age $30-75$ years; 2 ) sinus rhythm at ECG; 3 ) lack of history of chronic kidney disease; 4) no history of using antihypertensive drugs used or stopped taking antihypertensive drugs for more than 2 weeks before the study; 4) LVEF (Simpson's) > 50\%; 5) finishing ABPM test. The exclusion criteria included the following: 1 ) secondary hypertension; 2 ) myocardial infarction; 3) myocarditis. 4) structural heart disease; 5) peripheralartery disease; 6) chronic kidney disease; 7) autoimmune disease or tumor.

All experimental data were collected from the database of the medical center and recorded by two authors (Fu and $\mathrm{Hu}$ ).

\section{Blood pressure and laboratory measurements}

Office BP measurements in the clinic were performed by experienced community doctors and nurses using a calibrated OMRON Upper Arm Electronic Sphygmomanometer (Model HBP1100U). After the participants had taken a seat and rested for at least 5 minutes, the cuff blood pressure measurement obtained three consecutive readings that were averaged for the analysis. ABPM was performed uniformly using the verified and qualified TM2430 Oscillometric Ambulatory Blood Pressure Monitor of AND Company. The participants' blood pressure was continuously monitored for 24 hours, for every 20 minutes during the waking period and every 30 minutes during the sleeping period. Unqualified data (including less than $70 \%$ success rate of blood pressure measurement or $<20$ readings during waking period or $<7$ readings during sleep period) were repeatedly measured. According to the 2013 European Society of Hypertension (ESH) criteria [6], MHT was defined as office blood pressure (average of three readings) $<140 / 90 \mathrm{mmHg}$, but ambulatory blood pressure 24 -h average blood pressure $\geq 130 / 80 \mathrm{mmHg}$ and/or daytime (waking period) average blood pressure $\geq 135 / 85 \mathrm{mmHg}$ and/or nighttime (sleeping period) average blood pressure $\geq 120 / 70 \mathrm{mmHg}$. Otherwise, non-hypertension (NHT) was considered.

Low-density lipoprotein cholesterol (LDL-C), uric acid, andcreatinine were detected using a BackmanAU5800 spectrophotometer via colorimetry orimmunoturbidimetry.

\section{Echocardiographic analysis}


A comprehensive transthoracic echocardiography examination was performed by experienced sonographers using the Vivid S6 M4S-RS Probe (GE Medical System) interfaced with a 2.5- to 3.5-MHz phased-array probe. With the subject in the left decubitus position and breathing normally, the observer obtained images and synchronized ECG signals from the apical four-chamber and two-chamber long-axis views along the parasternal long and short axes. All records included at least three cardiac cycles and were digitally stored for offline analysis. All recordings and measurements were recorded in accordance with the guidelines of the American Society of Echocardiography $[7,8]$. Strain represented the deformation of the myocardium and was used to describe the local shortening of the myocardium. It was defined as the percentage of change in the length of the myocardial fibers relative to the original length. When $L_{t}$ represented the myocardial length of a certain myocardial segment in a certain direction at the time of the cardiac cycle, and $L_{0}$ represented the initial length, strain $(S)$ was expressed by the formula:

$\mathrm{S}=\Delta \mathrm{L} / \mathrm{L}_{0}=\left(\mathrm{L}_{\mathrm{t}}-\mathrm{L}_{0}\right) / \mathrm{L}_{0}$.

Therefore, the calculation method of GLS was derived. On two-dimensional echocardiography, the peak GLS describes the relative length change of the left ventricular myocardium between end-diastole (MLd) and end-systole (MLs) [9]:

GLS (\%)=(MLs-MLd)/MLd.

Left ventricular dimensions (LVDd) and wall thickness, including interventricular septal wall thickness (IVS) and LV posterior wall thickness (LVPW) in diastole (d),were made in the parasternal long axis with the M-mode cursor positioned just beyond the mitral leaflet tips, perpendicular to the long axis of the ventricle.

The mitral flow velocities were recorded with pulsed-wave Doppler with the sample volume placed at the tip of the mitral valve tips from theapical four-chamber view. Myocardial velocities were recorded using a standard pulsed-wave Doppler technique. Left ventricular (LV) diastolic function was assessed by mitral inflow $E$ and $A$ wave sand early diastolic velocity e' at the septal and lateral mitral annulus as an indicator of LV relaxation $[10,11]$. LVEF was measured by Simpon's method. LV mass was calculated indirectly from the thickness of the ventricular wall:

LV mass $(g)=\left\{1.04 *(I V S d+L V D d+L V P W d)^{3}-(L V D d)^{3}\right\} * 0.8+0.6$

Body surface area (BSA) was expressed by:

$\mathrm{BSA}\left(\mathrm{m}^{2}\right)=0.0061 \times$ height $(\mathrm{cm})+0.0128 \times$ weight $(\mathrm{kg})-0.1529$.

LV mass index (LVMI) was calculated as:

$\mathrm{LVMI}=\mathrm{LV}$ mass/BSA.

\section{Statistical analysis}


Statistical analysis was performed in three steps. First, the baseline characteristics of the participants divided into two groups (MHT and NHT) were measured based on the following principles: (1)continuous variables were expressed as the means \pm standard deviations (for normal distribution) or medians/quartiles (for skewed distribution), and categorical variables were shown as the frequencies with percentages; (2) ttestfor normally distributed data, Mann-Whitney Utest for skewed distribution data, and chi-square test/Fisher's exact test for categorical variables were used to identify significant differences between the groups. Next, univariate and multivariate analyses of common hypertensionrelated factors and GLS were conducted to evaluate their effect on MHT. Then, the interaction analysis was conducted to assess whether the relation between GLS and MHT was affected by other factors. Finally, all hypertension-related factors were pooled for multivariate adjustment by logistic analysis and used to assemble generalized additive models (GAM) to identify relationship between GLS and MHT. If an incremental effect model was present, the inflection point of the curve was taken as the threshold for stratification. The inter-observer and intra-observer variabilities were evaluated by the intraclass correlation coeffificients (ICCs).

Comparisons with $\mathrm{P}<0.05$ (two-sided) were considered to be statistically significant. All analyses were performed with Stata 15.0, R(version 3.4.3) and EmpowerStats (http://www.empowerstats.com, X\&Y Solutions, Inc., Boston, MA).

\section{Results}

\section{Baseline characteristics}

Descriptive characteristics of the study population are shown in Table 1dichotomized by the presence or absence of MHT. The study included 308 consecutive participants, representing a range of community populations. The mean age was 57 years in the NHT group and 59 years in the MHT group. BMI, uric acid and smoking status were significantly different between the two groups.

\section{Echocardiographic and imaging parameters}

Table 2 lists the M-mode,2-dimentionaland speckle-tracking echocardiographic parameters of the study participants. The MHT group showed thicker IVSd $(8.99 \pm 0.98$ vs $10.01 \pm 1.37, \mathrm{P}<0.001)$ and LVPWd $(8.97 \pm 0.99$ vs $10.00 \pm 1.25, P<0.001)$. LV diastolic function indicators, such as $E / A(1.01 \pm 0.26$ vs 0.86 $\pm 0.23, \mathrm{P}<0.001)$ and $\mathrm{E} / \mathrm{e}^{\prime}(8.58 \pm 2.02$ vs $11.70 \pm 3.37, \mathrm{P}<0.001)$, were reduced in the MHT group while those of the NHT group were basically normal. LVMI was significantly enlarged in the MHT group (80.91 \pm 15.20 vs $97.82 \pm 20.46, P<0.001)$, while GLS was lower ('worse') $(-15.79 \% \pm 2.81 \%$ vs $-19.62 \% \pm 1.67 \%$, $\mathrm{P}<0.001$; Fig. 1). Besides, LVEF was not significantly different between the groups. 
Table 1

Baseline characteristics of the study population.

\begin{tabular}{|c|c|c|c|}
\hline & NHT & MHT & P-value \\
\hline$N$ & 168 & 140 & \\
\hline Age & $57(49-64)$ & $60(55-65)$ & 0.085 \\
\hline BMI & $23.2 \pm 2.5$ & $24.4 \pm 3.4$ & $<0.001$ \\
\hline Male & $81(48.2 \%)$ & $60(42.9 \%)$ & 0.347 \\
\hline LDL-C, mg/dL & $92.4 \pm 21.4$ & $97.3 \pm 23.4$ & 0.056 \\
\hline Uric acid, $\mathrm{mg} / \mathrm{dL}$ & $335.3(293.6-393.4)$ & $391.7(318.9-476.5)$ & $<0.001$ \\
\hline Diabetes & & & 0.260 \\
\hline No & $150(89.3 \%)$ & $119(85.0 \%)$ & \\
\hline Yes & $18(10.7 \%)$ & $21(15.0 \%)$ & \\
\hline Smoking & & & 0.036 \\
\hline No & 147 (87.5\%) & $110(78.6 \%)$ & \\
\hline Yes & $21(12.5 \%)$ & $30(21.4 \%)$ & \\
\hline Alcohol & & & 0.333 \\
\hline No & $167(99.4 \%)$ & 137 (97.9\%) & \\
\hline Yes & $1(0.6 \%)$ & $3(2.1 \%)$ & \\
\hline \multicolumn{4}{|c|}{ Values are shown as mean \pm SD or mean (Q1-Q3) or $n(\%)$} \\
\hline $\begin{array}{l}\text { NHT: Non-Hyperte } \\
\text { Lipoprotein Chole }\end{array}$ & IHT: Masked Hyperte & Body Mass Index; LD & ensity \\
\hline
\end{tabular}


Table 2

Echocardiographic data of the study population.

\begin{tabular}{|c|c|c|c|}
\hline & NHT & MHT & P-value \\
\hline N & 168 & 140 & \\
\hline LVDd, mm & $43.10 \pm 3.23$ & $44.12 \pm 4.46$ & 0.021 \\
\hline IVSd, mm & $9.0(8.0-9.5)$ & $10.0(9.0-11.0)$ & $<0.001$ \\
\hline LVPWd, mm & $9.0(8.0-9.5)$ & $10.0(9.0-11.0)$ & $<0.001$ \\
\hline LVEF, \% & $69.27 \pm 4.30$ & $69.73 \pm 6.23$ & 0.438 \\
\hline$E / A$ & $1.0(0.8-1.2)$ & $0.8(0.7-1.0)$ & $<0.001$ \\
\hline LVMI, g/m² & $79.8(72.2-87.0)$ & $95.4(81.5-111.8)$ & $<0.001$ \\
\hline$E / e^{\prime}$ & $8.3(6.8-10.0)$ & $11.7(9.0-14.0)$ & $<0.001$ \\
\hline GLS, \% & $-19.9(-20.5--18.5)$ & $-15.2(-17.4--13.6)$ & $<0.001$ \\
\hline \multicolumn{4}{|c|}{ Values are shown as mean \pm SD or mean (Q1-Q3). } \\
\hline \multicolumn{4}{|c|}{$\begin{array}{l}\text { NHT: Non-Hypertension; MHT: Masked Hypertension;LVDd:Left Ventricular Diastolic Dimension; IVSd } \\
\text { Interventricular Septal Depth; LVPWd: Left Ventricular Posterior Wall Depth; LVEF:Left Ventricular } \\
\text { Ejection Fraction; E/A:E peak/A peak; LVMI: Left Ventricular Mass Index; E/e': E peak/e' peak; GLS: } \\
\text { Global Longitudinal Strain. }\end{array}$} \\
\hline
\end{tabular}

As shown in Table 3, GLS was significantly associated with MHT in both univariate (OR: 1.97, $\mathrm{P}<0.001)$ and stepwise multivariate regression analysis (OR: 1.99, P<0.001). In the existing and exploratory subgroups of the research entity, no interaction between the various factors was observed, which demonstrated that the relation between GLS and MHT was consistently significant (Supplementary Table 1). 
Table 3

Association of MHT and related factors in the study subjects.

\begin{tabular}{|lllll|}
\hline & Univariate & \multicolumn{3}{l|}{ Multivariate } \\
\hline Males & OR & $\mathrm{P}$ & OR & $\mathrm{P}$ \\
\hline Age & 0.81 & 0.348 & 0.60 & 0.173 \\
\hline BMI & 1.02 & 0.088 & 1.03 & 0.104 \\
\hline LDL-C & 1.15 & 0.001 & 0.91 & 0.155 \\
\hline UA & 1.01 & 0.057 & 1.02 & 0.013 \\
\hline Diabetes & 1.01 & $<0.001$ & 1.01 & 0.002 \\
\hline Smoking & 1.47 & 0.262 & 0.75 & 0.591 \\
\hline Alcohol & 1.01 & 0.038 & 1.90 & 0.217 \\
\hline LVMI & 3.66 & 0.264 & 0.62 & 0.725 \\
\hline GLS & 1.06 & $<0.001$ & 1.05 & $<0.001$ \\
\hline BMI: Body Mass Index; LDL-C:Low-density Lipoprotein Cholesterol; UA:Uric Acid; LVMI: Left Ventricular & \\
\hline Mass Index; GLS: Global Longitudinal Strain. & $<0.001$ & 1.99 & $<0.001$ \\
\hline
\end{tabular}

The cutoff point in nonlinear trend between MHT and GLS

After adjusting for individual and clinical factors of $\mathrm{MHT}$, we obtained the nonlinear correlation between MHT and GLS (Fig. 2). People without MHT had GLS below - 20\%, and when GLS was greater than $20 \%$, the potential risk of MHT gradually increased. The threshold of GLS detectable myocardial damage caused by diagnosed MHT was $-14 \%$.

Illustration: The x-axis shows GLS, and the $y$-axis shows the incidence of MHT, with the dotted lines area representing 95\% confidence intervals(Linear trend: $P<0.001)$.

\section{Interobserver and Intraobserver Variability}

As it has been shown in Table 4, inter-observer and intra-observer correlation were high for GLS measurement. 
Table 4

Intra- and inter-observer variability.

\begin{tabular}{|lccc|}
\hline & ICC & $95 \% \mathrm{Cl}$ & $\mathbf{P}$ \\
\hline Intraobserver variability & 0.993 & $0.991-0.994$ & $<0.001$ \\
\hline Interobserver variability & 0.956 & $0.945-0.964$ & $<0.001$ \\
\hline ICCs, intraclass correlation coeffificients; $\mathrm{Cl}$, confifidence interval. \\
\hline
\end{tabular}

\section{Discussion}

In the present study, we used GAM to elucidate the non-linear relationship between MHT and GLS among participants. As the probability of MHT arises, GLS gradually decreases. In the fully adjusted model, the $-14 \%$ of GLS was considered the critical value to represent absolute myocardial damage caused by MHT.

First, we evaluated the relationship between MHT and GLS through univariate regression analysis, and further confirmed the relation by multivariate regression after adjusting for the influence of the confounding factors. Since a previous study suggested that LVMI had a reduced GLS [12], we also adjusted LVMI for analysis. Next, in order to identify the mutual influence between the related factors, we conducted an interaction analysis. The results indicated that MHT and GLS had a strong relation in various situations. Finally, since GLS was a continuous variable, we plotted the fitting curve based on the dose-response relationship and found a nonlinear trend. With the help of the inflection point of the curve, we identified the risk threshold of GLS based on different MHT risk levels. To the best of our knowledge, this is the first description of the non-linear relation between MHT and GLS in a community-dwelling population.

Pickering et al. proposed the concept of MHT and emphasized the importance of identifying patients with MHT [13]. Since then, many studies have been done on the prognosis of MHT. A substudy of PAMELA cohort focusing on MHT patients showed that after follow-up for 10 years, the risk of new-onset left ventricular hypertrophy was high even after adjusting for confounding factors (OR: 2.22, $P=0.025$ ), emphasizing that this phenomenon represents the risk of heart damage with a poor prognosis [14]. The damage of MHT occurs at early stage as it elongates the longitudinal fibers, thickens myocardial fibers and ventricular wall, and remodels myocardium due to long-term increased afterload [15]. Compared with persistent hypertension, the attack of $\mathrm{MHT}$ is more insidious and poorly controllable; therefore, early recognition of MHT-related cardiomyocyte damage before heart failure is extremely important.

Two-dimensional (2D) speckle-tracking echocardiography technique, which can quantify myocardial strain by GLS measurement, has been validated by tagged CMR imaging and widely considered to be able to early detect myocardial damage even if the LVEF is preserved in various lesions[16-18]. Pathophysiologically, high levels of end-systolic wall stress, hypertrophic myocardium, and increased left ventricular stiffness play an important role in reducing the shortening of longitudinal subepicardial 
myocardial fibers, in accordance with the mechanism of cardiac impairment by MHT [19]. The latest guidelines also suggests that for patients with near-normal EF, global longitudinal strain has shown prognostic value, while prognostic information from EF is relatively less useful[20]. Santos et al. compared baseline GLS in 91 patients with pre-hypertension (systolic blood pressure 120-139 mm Hg or diastolic blood pressure 80-89 mm Hg, and not taking antihypertensive medication) and 105 patients with stage I hypertension (BP 140-159 or 90-99 mm Hg, and no current use of BP-lowering medication); the result showed that stage I hypertension already has an impaired GLS accounting to about $1 \%$ [21]. Although the $1 \%$ difference in GLS appears insignificant, studies have shown that a small decrease in GLS is a powerful indicator of hospitalization for heart failure, cardiovascular death, or cardiac arrest [22]. In terms of GLS evaluation in MHT, Tadic, et al. previously retrospected $56 \mathrm{MHT}$ patients and found that longitudinal strains was progressively deteriorated from normotensive group, particularly in the endocardial and midmyocardial layers [23]. Luo, et al. studied 40 young MHT patients and also concluded the similar results [24]. Both two studies compared the GLS difference between MHT and NHT, confirming the role of GLS in the assessment of cardiac function in MHT, but the correlation between MHT and GLS as well as potential cut-off value were not well defined. Our study included more MHT patients and strengthened the lower GLS in MHT than NHT by excluding disturbance or interaction from confounding factors. More importantly, we described the dose-response relationship and defined a critical value of $-14 \%$ as MHT-related myocardial damage. One explanation for this trend is that the pathology of MHT analogous to the early damage of hypertension, the changes of extracellular matrix and myocardial fibrosis mainly affect the subendocardium. Longitudinal fibers are more prone to fibrosis and hemodynamic overload due to their prominent subendocardial position. The more fibers damage, the less GLS. Therefore, based on this special relation, turning to early identification of MHT-related reversible cardiac damage by means of GLS may be a better way to discern potential victims and make further treatment decision.

Besides, similar to previous studies, lower GLS is also associated with lower mitral valve annulus diastolic velocity (E/A and E/E'), lower LVEF, more LVPWd and LVM, which represents the relationships among shortened systolic, impaired diastole, and advanced LV remodeling [24-25].

Our research also has limitations. First, the single-center retrospective study design prevents us from detecting causal relationships. Second, with the fact that the participants were from one community with unique population characteristics, life style and dietary culture, the extrapolation of our conclusions is limited. Finally, further follow-up studies are needed to explore whether the decrease in GLS in MHT patient group is associated with adverse events.

\section{Conclusion}

The relationship between MHT and GLS among participants is non-linear after adjusted for other relative risk factors, and the $-14 \%$ of GLS is considered to represent the cut-off point for absolute myocardial damage caused by MHT. 


\section{Declarations}

\section{Author Contributions}

Ming Fu and Xiangming Hu contributed to the data collection and drafting of the manuscript. Shuo Sun and Shixin Yi conducted the data analysis. Ying Zhang and Yingqing Feng contributed to the acquisition of the data. Yingling Zhou and Qingshan Geng reviewed and made critical revisions to the manuscript. Haojian Dong contributed to the ideas and approved the final version of the manuscript. All authors read and approved the final manuscript.

\section{Acknowledgement}

We thank LetPub for language editing assistance.

\section{Funding}

Our research was supported by The National Key Research and Development Program of China (No. 2016YFC1301202).

\section{Conflict of interest}

The authors declare that they have no competing interests.

\section{References}

1. Peacock J, Diaz KM, Viera AJ et al (2014) Unmasking masked hypertension: prevalence, clinical implications, diagnosis, correlates and future directions. J Hum Hypertens 28:521-528

2. Booth JN 3rd, Diaz KM, Seals SR et al (2016) Masked Hypertension and Cardiovascular Disease Events in a Prospective Cohort of Blacks: The Jackson Heart Study. Hypertension 68(2):501-510

3. Zhang DY, Guo QH, An DW et al. A comparative meta analysis of prospective observational studies on masked hypertension and masked uncontrolled hypertension defined by ambulatory and home blood pressure (2019) J Hypertens 37:1775-1785

4. Anstey DE, Pugliese D, Abdalla M et al (2017) An Update on Masked Hypertension. Curr Hypertens Rep 19(12):94

5. Aronow WS (2017) Masked hypertension. Ann Transl Med 5(23):456

6. O'Brien E, Parati G, Stergiou G et al (2013) European Society of Hypertension Working Group on Blood Pressure Monitoring. European Society of Hypertension position paper on ambulatory blood pressure monitoring. J Hypertens 31:1731-1768

7. Lang RM, Bierig M, Devereux RB et al. Recommendations for chamber quantification: a report from the American Society of Echocardiography's Guidelines and Standards Committee and the Chamber Quantification Writing Group, developed in conjunction with the European Association of 
Echocardiography, a branch of the European Society of Cardiology J Am Soc (2005) Echocardiogr 18:1440-1463

8. Nagueh SF, Smiseth OA, Appleton CP et al (2016) Recommendations for the evaluation of left ventricular diastolic function by echocardiography: an update from the American Society of Echocardiography and the European Association of Cardiovascular Imaging. J Am Soc Echocardiogr 29:277-314

9. Lang RM, Badano LP, Mor-Avi V et al (2015) Recommendations for cardiac chamber quantification by echocardiography in adults: an update from the American Society of Echocardiography and the European Association of Cardiovascular Imaging. J Am Soc Echocardiogr 28(1):1-39.e14

10. Sohn DW, Chai IH, Lee DJ et al (1997) Assessment of mitral annulus velocity by Doppler tissue imaging in the evaluation of left ventricular diastolic function. J Am Coll Cardiol 30(2):474-480

11. Ommen SR, Nishimura RA, Appleton CP et al (2000) Clinical utility of Doppler echocardiography and tissue Doppler imaging in the estimation of left ventricular filling pressures: A comparative simultaneous Doppler-catheterization study. Circulation 102(15):1788-1794

12. Soufi Taleb Bendiab N, Meziane-Tani A, Ouabdesselam S et al (2017) Factors associated with global longitudinal strain decline in hypertensive patients with normal left ventricular ejection fraction. Eur $\mathrm{J}$ Prev Cardiol 24(14):1463-1472

13. Pickering TG, Davidson K, Gerin W et al (2002) Masked hypertension Hypertension 40(6):795-796

14. Cuspidi C, Facchetti R, Quarti-Trevano F et al (2019) Incident Left Ventricular Hypertrophy in Masked Hypertension. Hypertension 74(1):56-62

15. Ishizu T, Seo Y, Kameda $Y$ et al (2014) Left ventricular strain and transmural distribution of structural remodeling in hypertensive heart disease. Hypertension 63(3):500-506

16. Amundsen BH, Helle-Valle T, Edvardsen T et al (2006) Noninvasive myocardial strain measurement by speckle tracking echocardiography: validation against sonomicrometry and tagged magnetic resonance imaging. J Am Coll Cardiol 47(4):789-793

17. Marcus KA, Mavinkurve-Groothuis AM, Barends M et al (2011) Reference values for myocardial twodimensional strain echocardiography in a healthy pediatric and young adult cohort. J Am Soc Echocardiogr 24(6):625-636

18. Luo XX, Fang F, Lee AP et al (2014) What can three-dimensional speckle-tracking echocardiography contribute to evaluate global left ventricular systolic performance in patients with heart failure? Int $J$ Cardiol 172(1):132-137

19. Lee WH, Liu YW, Yang LT et al (2016) Prognostic value of longitudinal strain of subepicardial myocardium in patients with hypertension. J Hypertens 34(6):1195-1200

20. Marwick TH, Gillebert TC, Aurigemma G et al (2015) Recommendations on the use of echocardiography in adult hypertension: a report from the European Association of Cardiovascular Imaging (EACVI) and the American Society of Echocardiography (ASE)†. Eur Heart J Cardiovasc Imaging 16(6):577-605 
21. Santos ABS, Foppa M, Bertoluci C et al (2019) Stage I hypertension is associated with impaired systolic function by strain imaging compared with prehypertension: A report from the prever study. $\mathrm{J}$ Clin Hypertens (Greenwich) 21(11):1705-1710

22. Shah AM, Claggett B, Sweitzer NK et al (2015) Prognostic Importance of Impaired Systolic Function in Heart Failure With Preserved Ejection Fraction and the Impact of Spironolactone. Circulation 132(5):402-414

23. Tadic M, Cuspidi C, Vukomanovic V et al (2016) Does masked hypertension impact left ventricular deformation? J Am Soc Hypertens 10(9):694-701

24. Luo XX, Zhu Y, Sun Y, et al. Does Masked Hypertension Cause Early Left Ventricular Impairment in Youth? Front Pediatr. 2018;6:167.

\section{Figures}




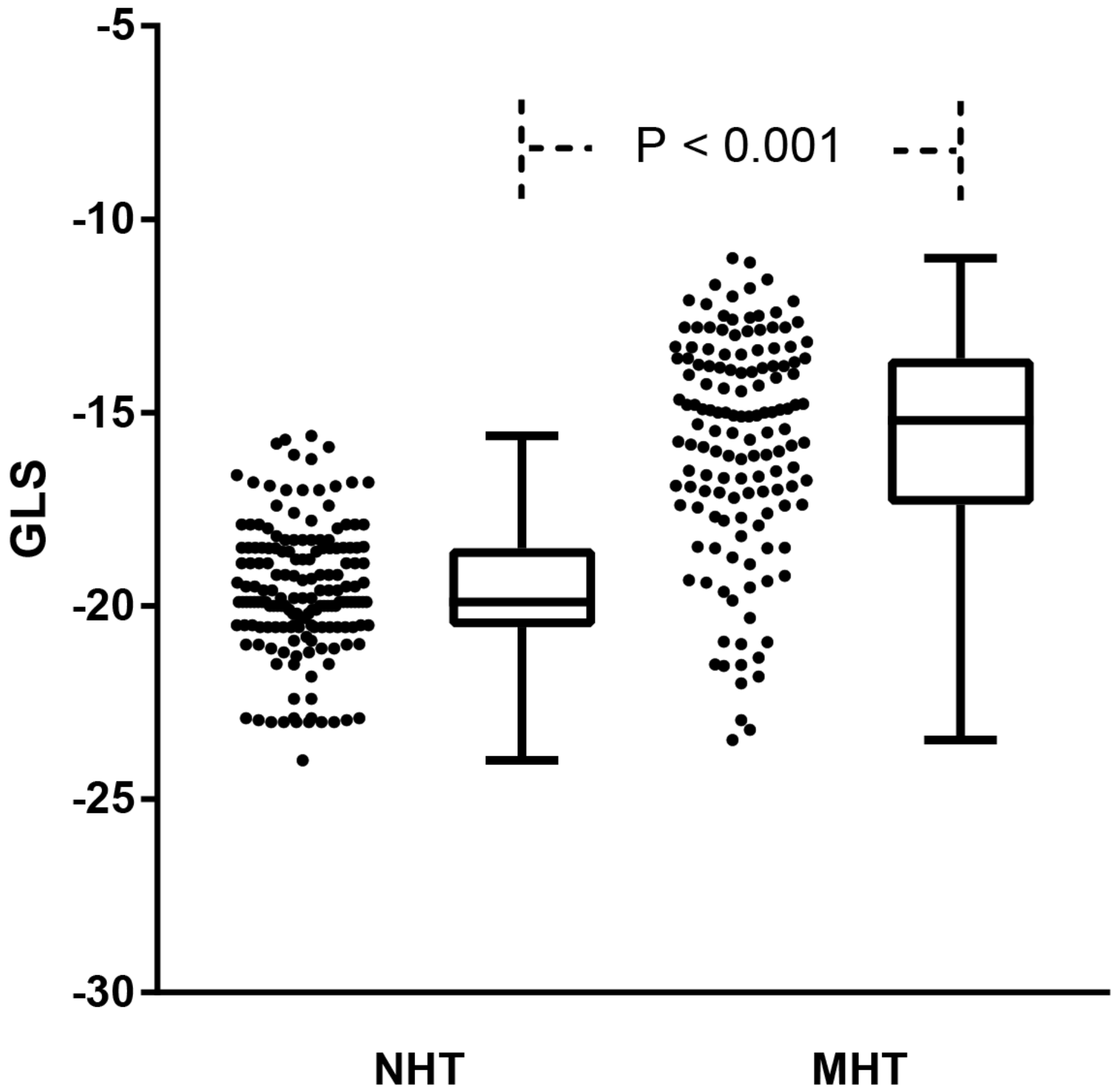

Figure 1

Bee chart of difference in GLS between NHT and MHT groups. 


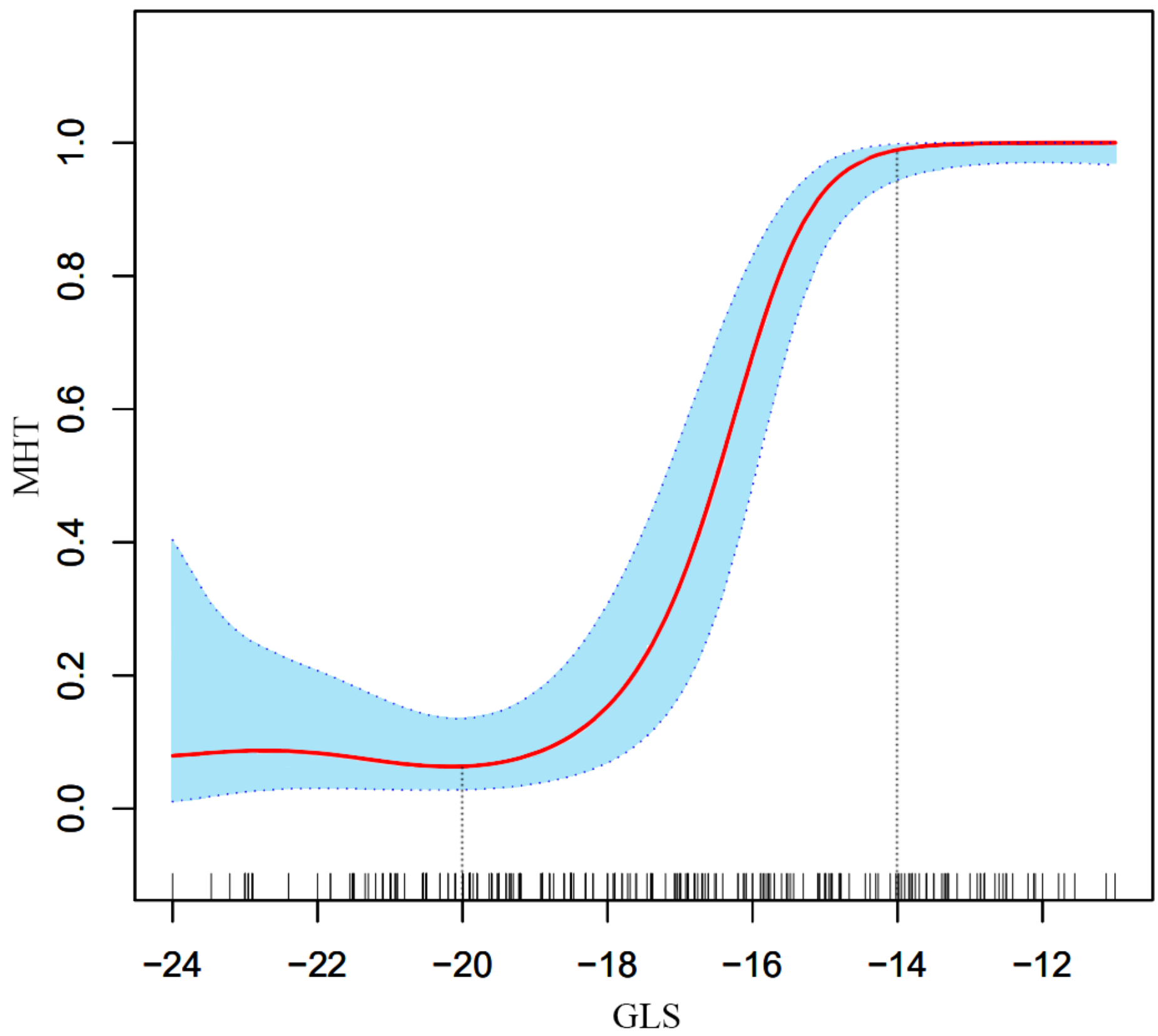

Figure 2

Non-linear relationship between GLS and MHT after adjustment 